\title{
Acute pancreatitis evoked by small-cell lung carcinoma metastases and detected by endoscopic ultrasound
}

Small-cell lung cancer (SCLC) is an aggressive disease that has a poor prognosis and tends to metastasize early [1]. The most frequent intra-abdominal sites of SCLC metastases include the adrenal glands and liver [2]. The pancreas is a rare site of SCLC metastasis. We report a case of acute pancreatitis triggered by SCLC metastasis in the pancreas.

A 52-year old woman with SCLC presented with vomiting and epigastric pain radiating to her back. Blood tests revealed increased alkaline phosphatase (ALP, $4.3 \mu \mathrm{kat} / \mathrm{L}$ ), gamma-glutamyl transferase (GT, $4.7 \mu \mathrm{kat} / \mathrm{L})$, and amylase $(5.2 \mu \mathrm{kat} / \mathrm{L})$, while bilirubin was normal. The patient had acute pancreatitis but no history of gallstone disease or excessive alcohol intake. A subsequent workup with transabdominal ultrasound showed no signs of disease in the liver, gallbladder, or pancreas, but a magnetic resonance cholangiopancreatography revealed dilatation of the extrahepatic bile duct $(9.5 \mathrm{~mm})$ and three unclear lesions in the pancreas, which raised suspicions of malignancy and led to a referral for endoscopic ultrasound (EUS).

EUS demonstrated three hypoechoic processes (two in the head and one in the body of the pancreas) with regular borders ( Fig. 1). The two processes (22 and $32 \mathrm{~mm}$ ) in the pancreatic head were compressing the pancreatic duct causing proximal dilatation of the duct $(4.5 \mathrm{~mm})$. Fine-needle aspiration (21-gauge needle) of one of the processes in the head of the pancreas followed by cytological examination revealed small-cell carcinoma cells ( $\bullet$ Fig. 2a) staining positively for thyroid transcription factor-1 ( Fig. 2b), which is a highly specific marker for primary lung adenocarcinomas.

The patient received a plastic biliary stent due to progressive cholestasis and continued abdominal pain. After recovery, the patient received palliative chemotherapy. In conclusion, we report an unusual case in which acute pancreatitis was induced by SCLC metastases. We point the focus on using EUS in the work-up of patients with acute pancreatitis of unknown aetiology.

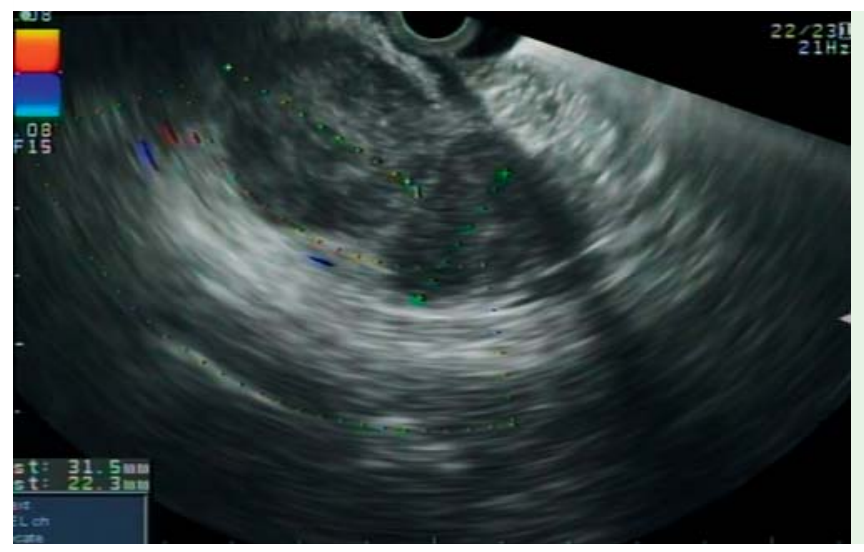

Fig. 1 Endoscopic ultrasound (EUS) image of the pancreas showing two hypoechoic processes with regular well-demarked borders in the head of the pancreas.
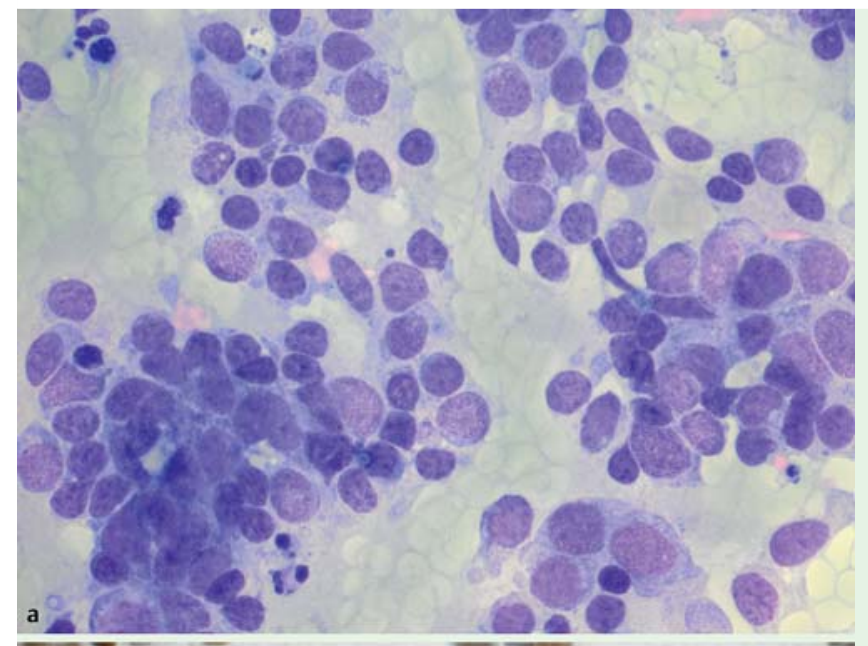

Fig. 2 Cytological examination following fine-needle aspiration of one of the processes in the pancreatic head revealed: a small cell carcinoma cells (single and loose clusters of small cells with a high nucleocytoplasmic ratio); b positive immunocytological expression of thyroid transcription factor- 1 .

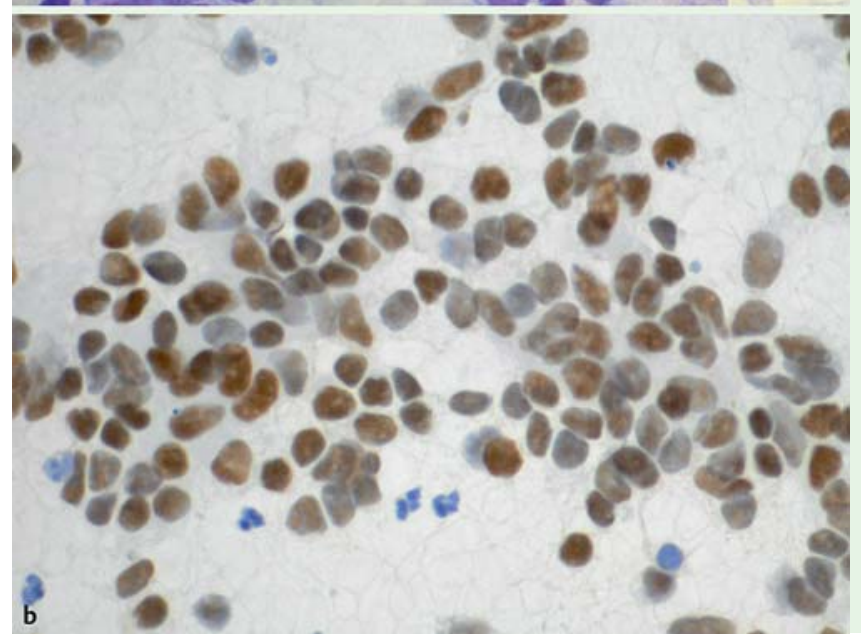


Endoscopy_UCTN_Code_CCL_1AF_2AZ_3AB

Competing interests: None

\section{G. Wurm Johansson', E. Toth', J. Torp ${ }^{2}$,} A. Ehinger ${ }^{3}$, L. Andersson ${ }^{2}$, H. Thorlacius ${ }^{4}$

${ }^{1}$ Section of Endoscopy, Department of Clinical Sciences, Malmö, Skåne University Hospital, Lund University, Malmö, Sweden

2 Department of Medicine, Central Hospital, Kristianstad, Sweden
${ }^{3}$ Section of Pathology, Department of Clinical Sciences, Malmö, Skåne University Hospital, Lund University, Malmö, Sweden

${ }^{4}$ Section of Surgery, Department of Clinical Sciences, Malmö, Skåne University Hospital, Lund University, Malmö, Sweden

\section{References}

1 Rodriguez E, Lilenbaum RC. Small cell lung cancer: past, present, and future. Curr Oncol Rep 2010; 12: 327-334

2 Jafri NF, Ma PC, Maulik G et al. Mechanisms of metastasis as related to receptor tyrosine kinases in small-cell lung cancer. J Environ Pathol Toxicol Oncol 2003; 22: 147-165
Bibliography

Dol http://dx.doi.org/

10.1055/s-0031-1291523

Endoscopy 2012; 44: E45-E46

(c) Georg Thieme Verlag KG

Stuttgart · New York

ISSN 0013-726X

\section{Corresponding author}

\section{H. Thorlacius, MD, PhD}

Section of Surgery

Department of Clinical Sciences, Malmö

Skåne University Hospital

Lund University

S-205 02 Malmö

Sweden

Fax: +46-40-336207

henrik.thorlacius@med.lu.se 